\title{
Arqueología histórica de Popayán \\ y la visibilización de su cultura tradicional
}

\author{
WILHELM LONDOÑO \\ UNIVERSIDAD DEL MAGDALENA \\ wilhelmlondono@gmail.com
}

\begin{abstract}
Resumen
[ste artículo habla sobre la cultura tradicional de Popayán, en especial sobre un [sentido de comunidad en el cual las diferencias sociales se producían en diálogo con una adscripción de toda la población a unos valores compartidos que posiblemente se reflejen en patrones de cultura material. Se presentan evidencias resultado de excavaciones arqueológicas en Popayán y su valle homónimo que se asocian con esa ideología.

PALABRAS CLAVE: historia colonial, cultura tradicional, arqueología histórica.
\end{abstract}

\section{A Historical Archaeology of Popayán, and His Traditional Culture Visibilization}

\begin{abstract}
This paper is about the traditional culture of Popayan, especially a sense of community in which social differences were produced in relationship with the adscription of whole population to some values that are represented in material culture. The article shows archaeological evidences from Popayán and its surrounding valley that are associated with these values.
\end{abstract}

KEY WORDS: colonial history, traditional culture, historical archaeology. 


\section{El Morro de Tulcán: MÁs QUE UNA PIRÁMIDE PREHISPÁNICA ${ }^{1}$}

\begin{abstract}
I. Este artículo no podría haber sido escrito sin el estímulo constante de Hernán Torres y Cristóbal Gnecco. Igualmente, la precisión en la exposición de las ideas no hubiera sido posible sin las sugerencias de los evaluadores anónimos del texto a quienes agradezco la gentileza de
\end{abstract}

$n$ la década de 1940 Julio César Cubillos (Cubillos, 1959) excavó - el Morro de Tulcán. En la publicación señaló que se trataba de Luna "pirámide prehispánica”. Dada su adherencia a una arqueología netamente descriptiva (cf. Gnecco, I995), Cubillos generó un sistema taxonómico que le permitía excluir de sus descripciones los materiales coloniales, que se sus comentarios. redujeron a unos cuantos fragmentos de loza vidriada. Como es bien sabido en arqueología (Goggin, 1968), el vidriado corresponde a una técnica que trata la superficie de las lozas adhiriéndoles una

capa de cuarzo y plomo, procedimiento que no se desarrolló en períodos prehispánicos (cf. Londoño, 2000).

Las investigaciones sobre la historia de la alfarería (López, 1977) han mostrado que el uso de plomo en el tratamiento de las superficies cerámicas evoca una técnica que se introdujo por los menos desde el siglo vi d. C. en la península ibérica, de tal suerte que su uso fue muy prolongado en esa región. En el momento de la Conquista de América los españoles introdujeron esa técnica sin que hasta el momento se hayan reportado los mecanismos de esa inclusión en lo que sería el Virreinato de la Nueva Granada.

Cubillos relegó los hallazgos poshispánicos a un apartado intitulado "Misceláneos". Igualmente, no configuró ningún nivel explicativo sobre el ánfora fusiforme y solo le bastó darle ese nombre para que la misma fuera así exhibida por más de cincuenta años con esa equivocada referencia (ver figura I).

En el año de 2004, en el marco del Tercer Congreso de Arqueología en Colombia, en una muestra cerámica que iba a ser botada por la Universidad del Cauca, el investigador Juan Guillermo Martín-Rincón reconoció fragmentos coloniales similares a otros desperdigados por toda la cuenca del Pacífico de Suramérica (cf. Rovira 1997, 2002). En ese momento se pensaba que esos materiales representaban mayólicas panameñas, cuando en realidad eran lozas producidas en el valle peruano de Moquegua (cf. Schávelzon, 200I). 
Volumen 47 (I), enero-junio 201

En el marco del mencionado reconocimiento, el catálogo de Kathleen Deagan (1987) y el de Monika Therrien et al. (2002) señalaron claramente que las ánforas cónicas con alto contenido de caolín era coloniales. Según el catálogo de Deagan, elaborado con base en los hallazgos de la Florida, el ánfora fusiforme del Morro del Tulcán correspondería, por la forma de su borde, a las botijas coloniales usadas a finales del siglo xviII, cuando la capacidad de contención se había aumentado.

$\mathrm{Al}$ analizar el contexto del hallazgo de Cubillos, es claro que el evento de deposición del ánfora se hizo con ejemplares de clara afiliación prehispánica caracterizados por el arqueólogo como pertene-

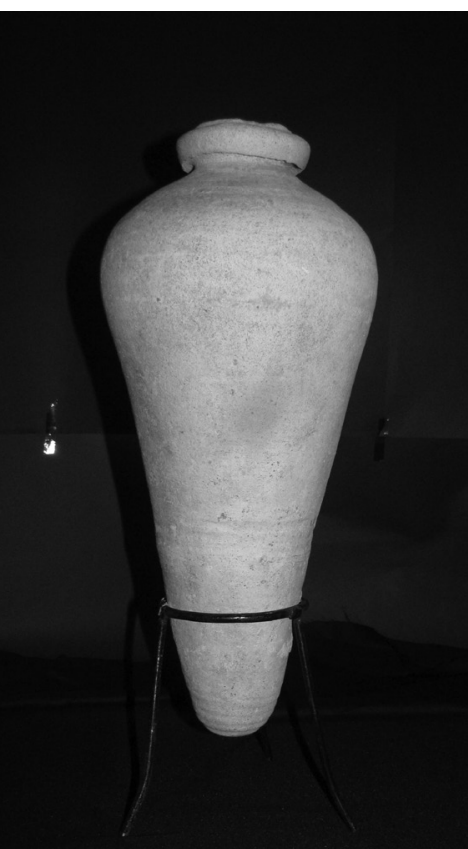

Figura I. Ánfora colonial del Morro de Tulcán Foto de Paola Sanabria. cientes a la tradición del formativo del suroccidente de Colombia (Cubillos, 1984), que, según la estimación, podía fecharse hace unos dos mil años. Ya que en el siglo xviII Popayán se había constituido en una importante ciudad de tránsito de mercaderías entre el Alto Perú y el puerto de Cartagena (cf. Barona, I995), era muy difícil que en esta época dejaran a los indígenas celebrar un enterramiento en predios de la propiedad de la familia Caldas (hoy el Morro de Tulcán conforma el límite norte del barrio Caldas que heredó el nombre de sus antiguos propietarios).

Lo que quedó claro con el análisis morfológico del ánfora era que la misma correspondía al período colonial. Tomando datos registrados para el Alto Perú (Rice, 1997, Rice \& Beck, I990; Rice \& Smith, 1989) fue posible comprender que ánforas similares eran fabricadas en los valles de Nazca, Ica y Pisco y en el sur de la región de Moquegua. En estas áreas, las investigaciones de Prudence Rice en la década de 1980 mostraron que la producción de vino fue una de las primeras industrias del siglo xvi. No solo se fabricaba vino, brandy y pisco, sino aceite de oliva y se produ- 
cían aceitunas que eran transportadas en estas ánforas que en la literatura colonial se denominaban botijas (cf. Brizuela, 2002).

Por las investigaciones de Prudence Rice en su proyecto "Bodegas de Moquegua" (Rice, 1997), se pudo demostrar que en esta región del Perú hubo dos grandes períodos de producción vinícola. El primero a finales del siglo xvi y el segundo a mediados del siglo XVIII. Según los datos de Rice, en el primer período se comenzó a producir botijas con una capacidad estándar de ocho litros. En el segundo período la capacidad casi se triplicó y llegó a un máximo de veintitrés litros por unidad. Según Rice, a mediados del siglo xviII más del 60\% del consumo de vino de la villa imperial de Potosí provenía de Moquegua; lo mismo ocurría en La Paz. En Oruro, más del 9I\% del consumo provenía de este valle peruano. Según los datos de archivo a los que tuvo acceso Rice, en Moquegua, para la década de I870, salían más de I.300.000 litros de brandy, lo que representaba más de 6.500.0oo litros de vino, pues para producir un litro de brandy se necesitaban cinco de vino. Según Rice, para el año de 1769, en la región de Escapalaque, en Moquegua, un productor reportó al fisco la producción de I3.715 botijas. Igualmente, declaró ser el administrador de otras 6.4I3 que pertenecían a los jesuitas.

Según lo que se puede apreciar en el extenso trabajo de archivo de Rice y en el excelente trabajo de Brown Kendall (I986) sobre la relación entre modernización y producción de brandy, Moquegua entregaba más del 90\% de la producción de vino a Potosí, Oruro, Cochabamba y La Paz y le alcanzaba para exportar un remanente a otras regiones. Ya que el interés de Rice y de Kendall no era el destino de la producción, no se sabe aún si en los archivos que ambos investigaron se habla explícitamente de Popayán o de otras regiones de la actual Colombia.

En la revisión archivística realizada por Alejandra Garcés para el proyecto sobre pautas de consumo de cerámica colonial y republicana ${ }^{2}$ (cf. Londoño, 2006),

2. Este proyecto fue financiado por la Fundación de Investigaciones Arqueológicas Nacionales (FIAN).

la documentación referente a la importación de bienes del Perú muestra claramente la introducción de botijas y pisco a Popayán en el siglo xviII. Como lo enseñan estos archivos, la cronología no tiene que ver directamente con el inicio de la actividad sino con los dispositivos fiscales configurados por el gobierno colonial para obtener remuneraciones 
Volumen 47 (I), enero-junio 201

de una actividad que se venía ejerciendo con anterioridad sin tributaciones controladas.

Aunque no se ha intentado una investigación más exhaustiva para datar la única botija completa hallada hasta ahora en Popayán, es muy probable que la misma sea del siglo xvi y corresponda al primer ciclo de producción de vino. Luego su contexto de deposición estaría hablando de la posibilidad de que las comunidades nativas del Valle de Popayán involucraran en matrices culturales prehispánicas bienes coloniales en un momento en que esas matrices aún no habían sido desmontadas.

En el excelente trabajo de Sergio Serulnikov (2006) sobre el mundo colonial andino, se hace evidente que en ciertos sectores del altiplano boliviano, por ejemplo, el régimen colonial permitió el ejercicio de vastas tradiciones nativas que convivieron con la episteme cristiana; estas eran ignoradas por el gobierno colonial, siempre y cuando esas comunidades tributaran con la frecuencia y cantidad requeridas. Ese fenómeno pudo ocurrir también en el Valle de Popayán hasta el siglo xviII, cuando la ciudad adquirió una fisonomía de enclave hispano más clara.

Según estas evidencias y como ya lo señaló Guido Barona (I995), Popayán, entendida como una ciudad con un efectivo control administrativo, con una burocracia estable y parcialmente eficaz, fue una producción del siglo xviII. Antes de ese período era una villa con un número pequeño de peninsulares que debían negociar con las elites indígenas su permanencia en la región. De allí que estas elites tuvieran acceso a las importaciones que se hacían del Alto Perú.

Esta serie de contextos arqueológicos híbridos apenas comienzan a ser intuidos para el Valle de Popayán y corresponden realmente no a una serie nueva de hallazgos sino a desarrollos teóricos recientes que tratan de ver de una manera diversa la cultura colonial apelando a documentación de archivo. Al igual que Cubillos, Gerardo Reichel-Dolmatoff también se enfrentó a fragmentos coloniales, y fue un poco más allá simplemente regalando esos tiestos al arqueólogo John Goggin (Goggin, 1968, p. 47).

En resumen, el Morro de Tulcán probablemente fue una pirámide cuyos inicios se remontan a períodos prehispánicos aún por determinar. Sin embargo, las evidencias que se pueden recoger de los artículos de Julio César Cubillos permiten inferir que los eventos deposicionales que él excavó eran netamente 
coloniales, tal vez referidos a inicios de la Colonia. Esto supone que en esa época las interacciones entre las comunidades nativas y los colonizadores eran más complejas y no se pueden explicar solo apelando a la documentación escrita. Si bien no se quiere decir con esto que no hubo un proceso de subordinación, este tiene que ser leído teniendo en cuenta el papel activo de las comunidades en la clave de resistencias no solo abiertas sino cotidianas, tal como ha sido sugerido para otras partes de Colombia (Loboguerrero, 200I).

\section{LO QUE MUESTRA EL REGISTRO ARQUEOLÓGICO COLONIAL}

Com

omo se puede apreciar arriba, Gerardo Reichel-Dolmatoff y Julio César Cubillos, el primero desechando las lozas coloniales, el segundo describiéndolas pero sin adjudicarles horizontes analíticos, estaban cruzados por una lógica epistémica que hacía un repartimiento disciplinario de los órdenes fenomenológicos. Ellos, en tanto arqueólogos, se adjudicaban el estudio del pasado prehispánico. Axiomáticamente, reconocían en los historiadores el derecho de hablar sobre el período colonial. Salvo ReichelDolmatoff (cf. Reichel-Dolmatoff 1950, I951a, 1951b, 1953, 1954), el modus operandi de estos arqueólogos relegaba a los etnógrafos las investigaciones de comunidades contemporáneas.

Este repartimiento epistemológico puede ser nominado como la distribución estratigráfica de la cultura. En los horizontes más profundos están los arqueólogos como etnógrafos del pasado. En el nivel medio están los historiadores como agentes intermedios entre los arqueólogos y los antropólogos. Finalmente, los etnógrafos como descriptores de mundos premodernos en vísperas de desconfigurarse (Fabian, I983).

En el marco del proyecto mencionado arriba sobre la arqueología histórica de Popayán, esta lógica epistemológica denominada acá distribución estratigráfica de la cultura fue puesta en tensión. La tarea fundamental consistía en revisar el período colonial desde las evidencias materiales como si fueran las únicas fuentes. Sin duda alguna, esta era una tarea bastante iconoclasta respecto de la tendencia en las investigaciones arqueológicas en el Valle de Popayán hacia los períodos precerámicos. 
Con el propósito de conocer los procesos de consumo de cerámica colonial y republicana en el Valle de Popayán, nuestra primera tarea fue aproximarnos a los contextos coloniales de consumo de loza colonial en ámbitos indígenas, al igual que los espacios de consumo central de cerámica indígena (Londoño, 2006).

Tras este objetivo, se realizaron excavaciones arqueológicas en el poblado de Timbío, a unos diez kilómetros al sur de Popayán, como también en el resguardo nasa de Novirao a unos trece kilómetros al norte de Popayán. Igualmente, se analizó la información recolectada por Miguel Méndez en sus excavaciones en el centro histórico de Popayán (Méndez, 2007).

En el poblado de Timbío se excavaron dos terrazas de un complejo de unidades domésticas concentradas de lo que al parecer fue una aldea indígena. La selección de las áreas se tomó con base en dos criterios: por un lado, se seleccionó la terraza más grande, la cual se denominó Ti; por otro, se excavó una terraza adyacente con un tamaño modal del conjunto de terrazas que se denominó T2 (ver figura 2).

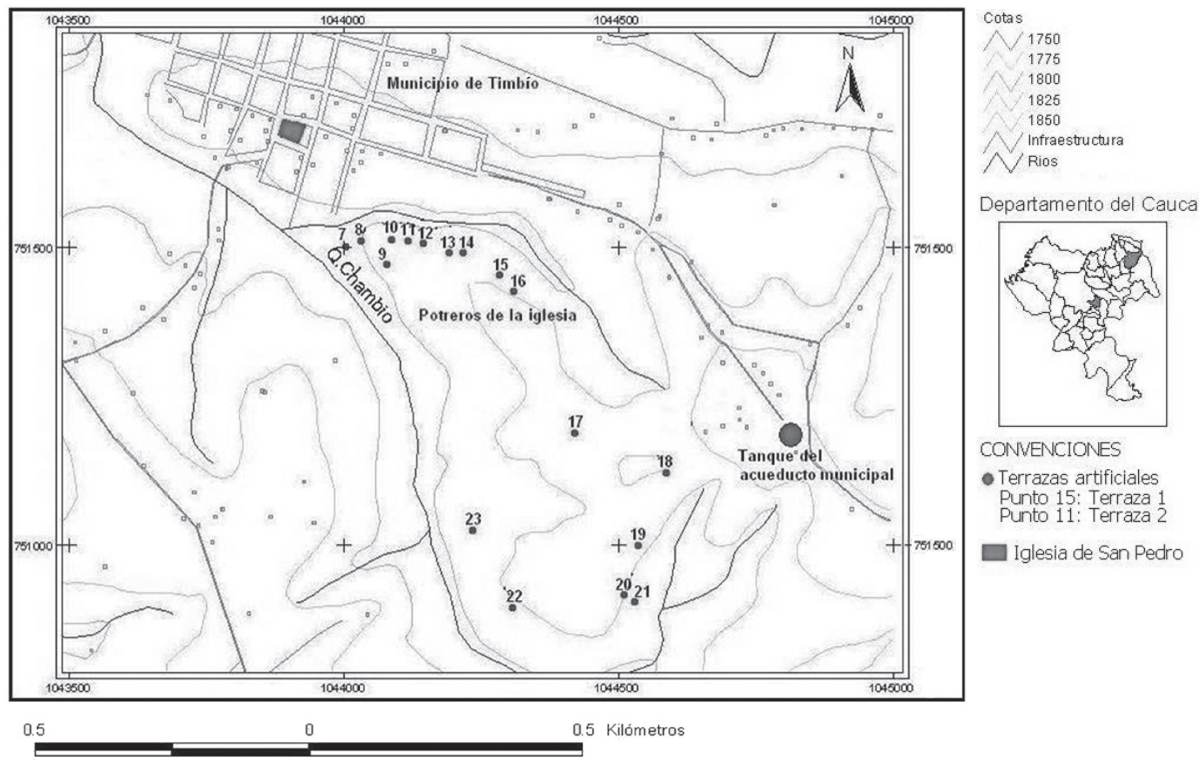

Figura 2. Mapa de Timbío

Fuente: Londoño (2006). 
Para darles una organización temporal y espacial a los hallazgos, se realizó una síntesis de las taxonomías cerámicas de la región. De tal suerte, se definieron dos grandes conjuntos cerámicos en los cuales la organización tecnológica evidenciaba las tradiciones coloniales o indígenas siguiendo la propuesta de Monika Therrien et al. (2002). Dentro del primer grupo se contabilizaron tres tipos cerámicos muy comunes en el suroccidente de Colombia, los cuales han sido asociados a los períodos formativos de esta región del área intermedia (cf. Cubillos, i984; Urdaneta, I99I). Estos son: Popayán Alisado sin Pintura, Popayán Baño Rojo y Popayán Inciso. Aunque no es este el lugar para entrar en una discusión de lo problemática que resulta esta periodización, esta tradición alfarera se ha asociado a los procesos sociopolíticos previos a la configuración de estratificaciones sociales (ReichelDolmatoff, 1997). En el segundo grupo se contabilizaron las lozas producidas en torno y esmaltadas con plomo o estaño, lo que corresponde a una tradición peninsular (Therrien et al., 2002). Hay una particularidad con este material y es que puede ser de fabricación local o importado. Dentro de los locales se encuentra una variedad importante de vidriados donde sobresalen tres colores importantes: el verde, el amarillo y el café. Dentro de las importadas hay dos tipos de cerámicas, las europeas y las mayólicas americanas (ver figura 3).

Un elemento que caracterizó todos los hallazgos del proyecto de arqueología histórica de 2006 fue que ni en Timbío, ni en Novirao, ni en Poblazón ni en Puelenje (áreas periféricas

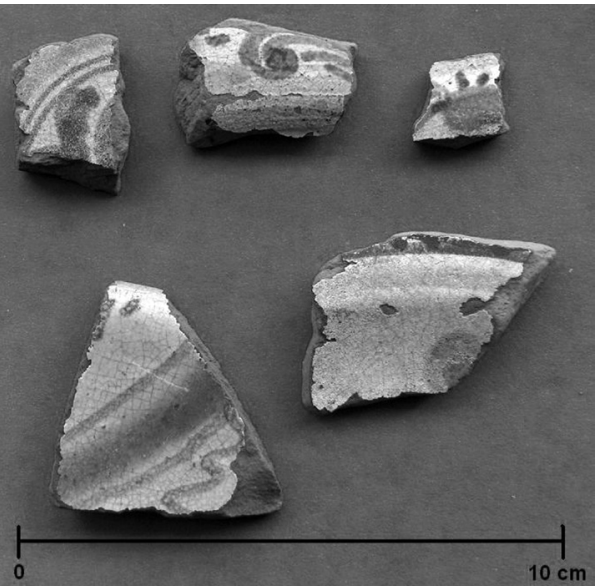
a finales de la Colonia e inicios de la República) se encontró cerámica colonial importada. En cambio, en el centro histórico se reportaron hallazgos de mayólica americana y europea.

En su trabajo arqueológico, Ana Caicedo (2006) excavó un sitio en el barrio El Empedrado en el centro

Figura 3: Mayólica americana de la colección de Miguel Méndez Fuente: Londoño (2006). 
histórico de Popayán. En su excavación, las mayólicas americanas aparecen en los últimos cuarenta centímetros contados desde la base compartiendo horizontes con fragmentos indígenas (alisados y engobados) (Caicedo, 2006, p. 26). Aunque no es el propósito llegar a un análisis detallado de los procesos de formación del registro arqueológico colonial, es importante mencionar que lo que señala esta excavación es que el compartir un mismo horizonte estratigráfico evoca eventos similares de deposición y tal vez de uso de esos artefactos.

Dentro del proyecto de arqueología histórica desarrollado en Popayán (Londoño, 2006) se hicieron algunas excavaciones en el centro histórico. Se pudo constatar la existencia en los horizontes más profundos de Mayólica Local (ML) y tipos indígenas (Popayán Alisado Sin Pintura, PASP; Popayán Baño Rojo, PBR) (ver figuras 4 y 5). En el estrato tres (ver figuras 4 y 5) se halló Mayólica Americana Polícroma que en ese momento de la investigación se referenció como Mayólica Panamá Polícroma (MPP). Lo interesante de este contexto es que las mayólicas locales y americanas estaban en los estratos más profundos, lo que da cuenta de su mayor antigüedad.

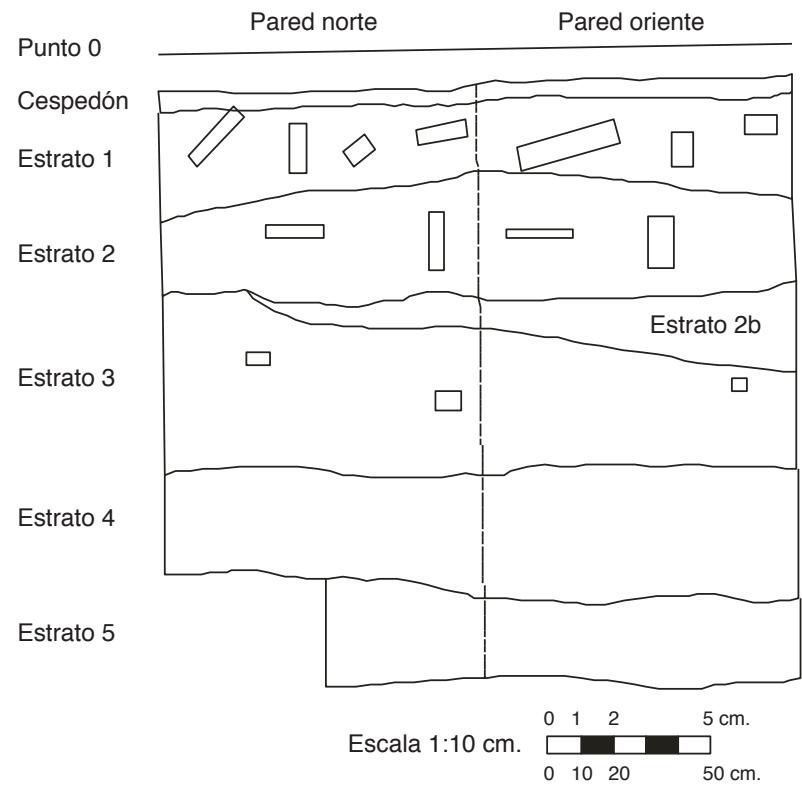

Figura 4. Estratigrafía del Hotel Familiar (centro histórico de Popayán) Fuente: Londoño (2006). 

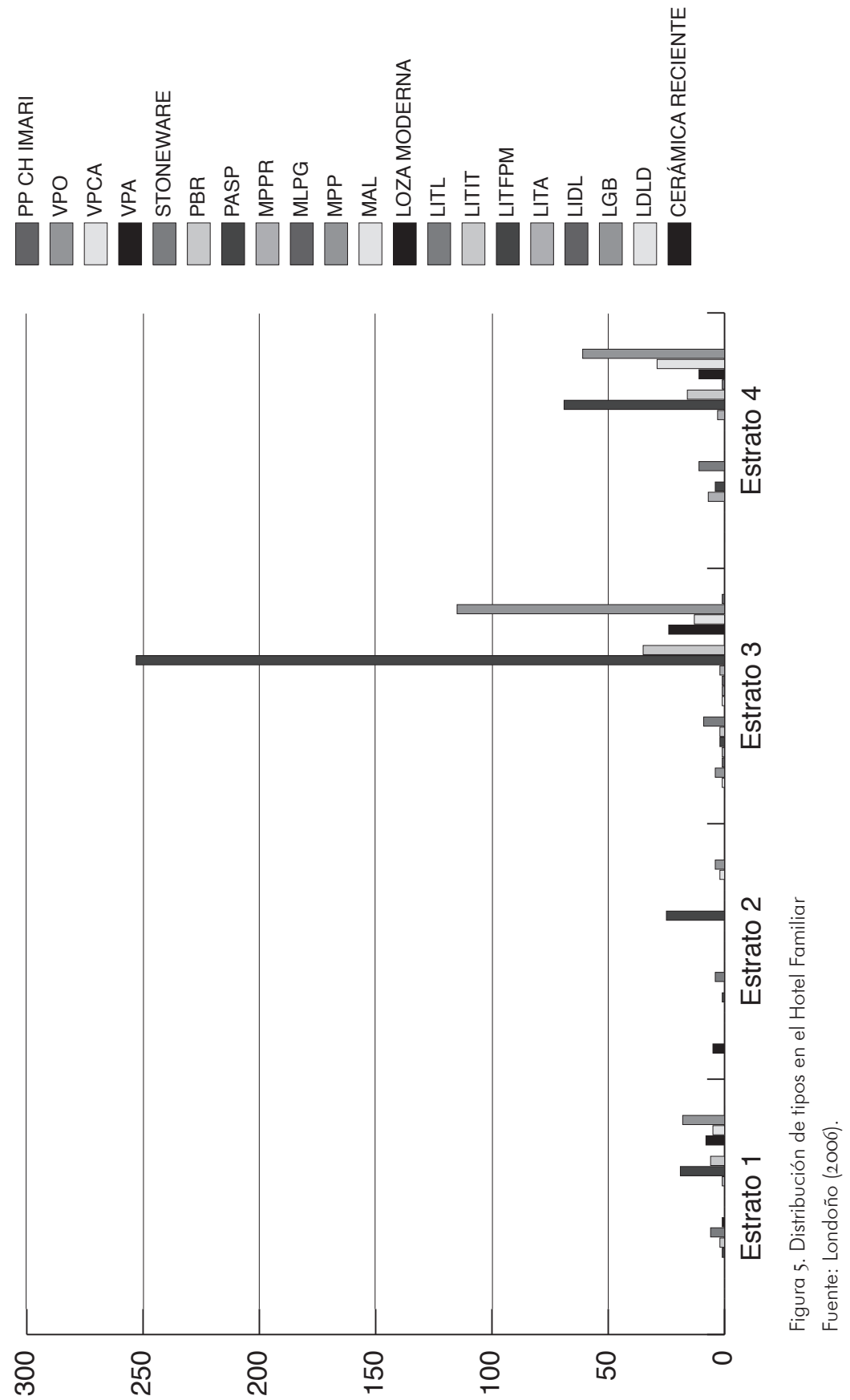
Para el caso de Panamá, se ha considerado que la Mayólica Panameña cesó de consumirse en el siglo XvII (cf. Rovira, 2002).

Revisada toda la documentación arqueológica disponible para el centro histórico de Popayán (Londoño, 2006), se puede apreciar una tendencia que consiste en que en los estratos más profundos, y por ende más antiguos, la mayólica americana aparece con tipos de tradición indígena como también con vidriados verdes. Esta tendencia después desaparece en los estratos más superficiales donde se encuentran las lozas industriales y más vidriados locales con nuevos colores y formas.

En el extremo sur del Valle de Popayán, en Timbío, no se encontraron fragmentos de mayólicas americanas. Por el contrario, se hallaron bajas frecuencias de loza vidriada local verde en los niveles superficiales. El tipo más frecuente fue el alisado indígena que se encontró a lo largo del horizonte de excavación. Aunque los hallazgos apenas arrojan pistas de lo que fue esa concentración demográfica, las evidencias señalan un alto consumo de cerámica indígena con muy bajas frecuencias de vidriado local verde (ver figura 6).

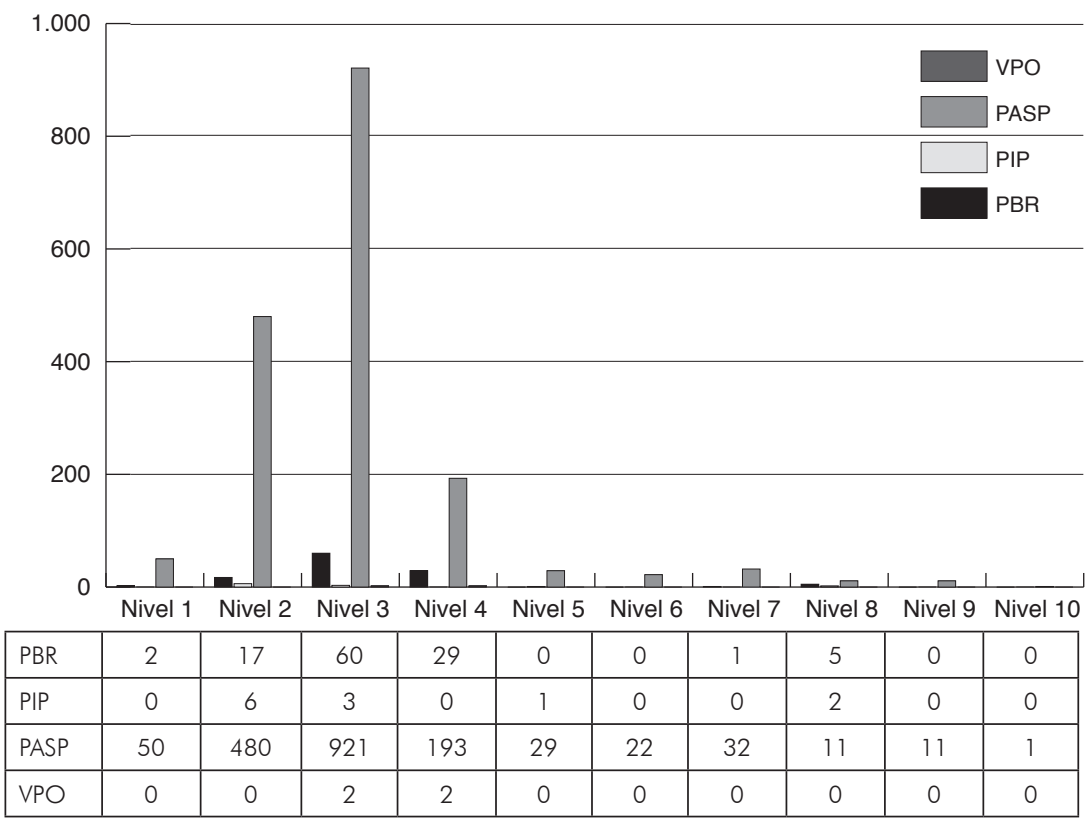

Figura 6. Distribución de TI (Timbío)

Fuente: Londoño (2006). 
A diferencia de Timbío, al norte del Valle, en el resguardo indígena Novirao, el registro señaló altas frecuencias de vidriados que combinan, además de los tonos oliva, amarillo y café. Otro de los elementos interesantes en Novirao fue la presencia de mayores formas, lo que señaló una correlación positiva entre la variedad cromática y funcional (Londoño, 2006). Es decir, que a medida que avanza la línea del tiempo aparecen vidriados de más colores y formas que comparten horizontes con lozas industriales (ver figura 7).

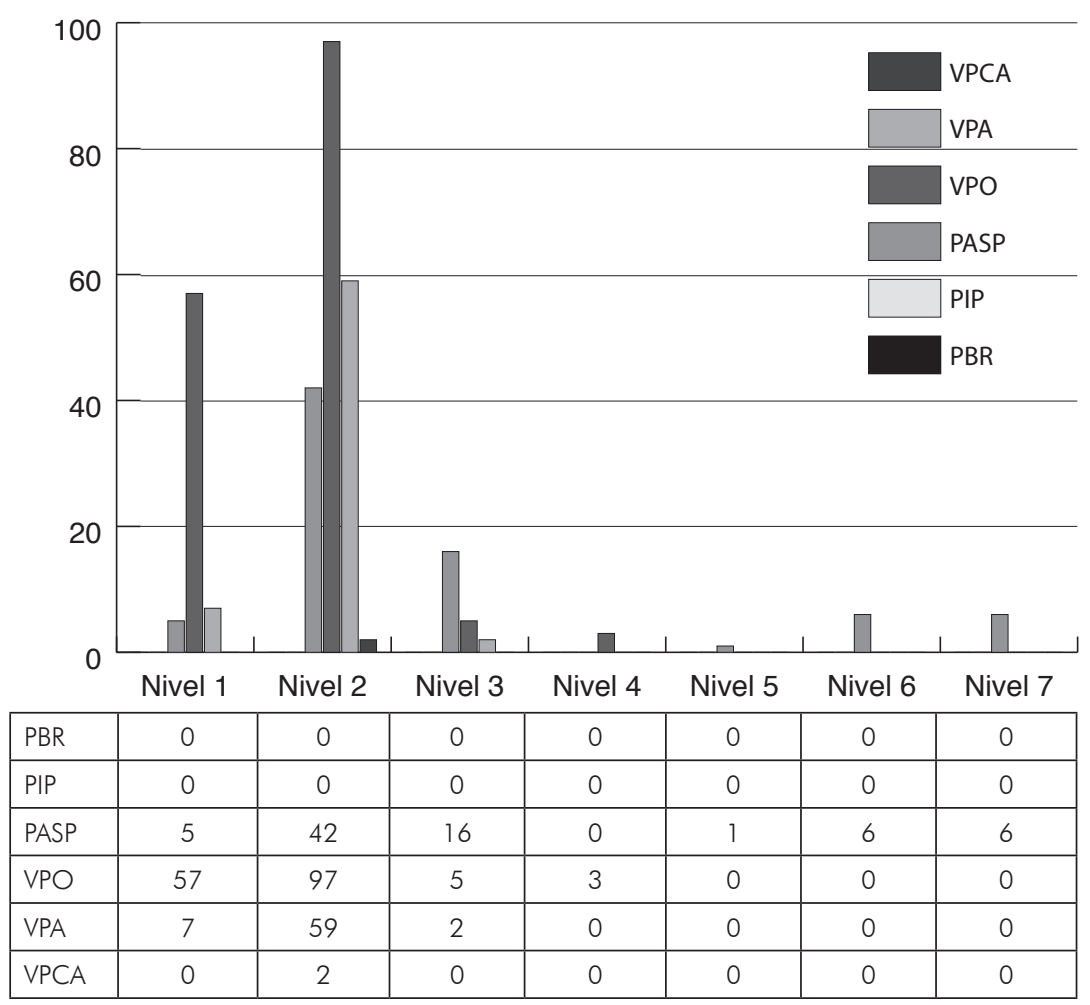

Figura 7. Distribución de tipos en Novirao Fuente: Londoño (2006).

En el caso de Novirao y de Poblazón, la correlación positiva mencionada arriba conlleva una asociación cronológica vinculada con las lozas industriales. Si bien estas lozas tienen un origen inglés (Therrien et al., 2002), hay evidencia de que para mediados 
Volumen 47 (I), enero-junio $20 I I$

del siglo xix se adquirió una fábrica de loza en Bogotá en la cual pudo producirse una importante suma de objetos que fueron consumidos en diversos espacios sociales. Con las evidencias que contamos se podría plantear la hipótesis de que estas lozas eran traídas de Bogotá a Popayán. Evidentemente eso debe ser corroborado. De tal suerte, los contextos de Poblazón y Novirao serían muy tardíos comparados con los de Timbío.

En relación con la cerámica que se encontró en el centro histórico hay dos cosas importantes que decir. En primer lugar, aparece como tipo predominante de la muestra el vidriado oliva. En un análisis que hizo Fabio Hernán Polo (2009), este encontró que el vidriado oliva de producción local es el que mayores diámetros tiene. Como base del análisis tomó todos los fragmentos de cerámica excavados durante más de treinta años de investigaciones arqueológicas en el Valle de Popayán, y que corresponden a una muestra que Miguel Méndez recuperó tras las reparaciones de los claustros religiosos de Popayán destruidos en el terremoto de 1983. En cuanto al predominio de los vidriados oliva, ya reseñado (cf. Londoño, 2006), también aparece como relevante el análisis que hace Polo de algunos fragmentos de vidriado que al parecer fueron elaborados por medio de la técnica indígena del enrollado (ver figura 8).

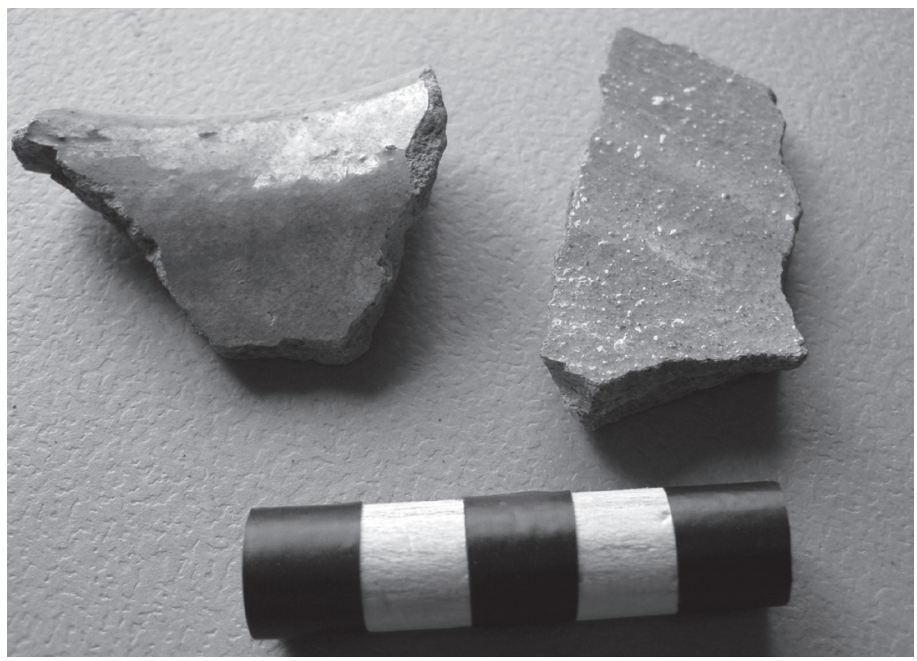

Figura 8. A la izquierda, Vidriado Amarillo elaborado con torno. Elaborado con rollos (nótese las acanaladuras)

Fuente: Londoño (2006). 
Aunque la elaboración de cerámica vidriada por medio de técnicas indígenas en el manejo de la arcilla es algo que apenas comienza a considerarse para la arqueología colonial del Valle de Popayán, es clara la existencia de fragmentos de tradición indígena, hallados en los claustros, con incisiones que representan la cruz potenzada.

Lo que muestra la evidencia hasta ahora recuperada es que las tradiciones de alfarería indígena en la Colonia siguieron produciendo artefactos que eran usados por las elites religiosas o seculares del centro histórico de Popayán, con marcas netamente hispanas. Esto permitiría formular la hipótesis de que estas tradiciones nativas se conservaron en la medida en que incorporaban signos de la cultura colonial. Sin embargo, ello debe ser evaluado mediante investigaciones precisas.

Los elementos de cultura material referenciados señalan claramente que en ciertos espacios se mantuvieron las diferencias de consumo relacionadas con la adscripción a un grupo social. Estas diferencias sociales son evocadas por fragmentos como las mayólicas americanas o europeas que solo se hallan en el casco viejo de Popayán. A propósito de los hallazgos, este tipo de loza tiene sus picos de consumo en el siglo xVIII (y parte del xVII), por lo que permite generar indicaciones cronológicas interesantes.

Asimismo, otros fragmentos de cultura material indican que las tecnologías indígenas se mantuvieron tanto en Popayán como en el Valle homónimo, lo cual se comprueba por la permanencia de tipos de cerámica burda, tal vez vinculada con procesos de cocción de alimentos, tanto en el casco viejo como en la periferia. Esta cerámica burda, que se reconoce por el color café de la pasta, se siguió consumiendo en el centro histórico y en los pueblos de indios, por lo menos hasta finales del xIx, cuando hacen su aparición las lozas de técnica inglesa y a mediados del $\mathrm{xx}$, cuando se introduce el aluminio.

En términos de los ajuares de las comitivas ${ }^{3}$, en el siglo XIX las mayólicas americanas son reemplazadas por lozas inglesas y

3. Las ilustraciones del siglo XVII y XVIII sobre las comidas familiares en la América española (Londoño, 2006) muestran que las mayólicas americanas y europeas se usaban para comer. Igualmente, la cultura material para la preparación de alimentos provenía de las tradiciones indias de alfarería. chinas en el centro de Popayán. En los pueblos de indios, al igual que en el centro de Popayán, aparecen vidriados locales de diversas formas y colores. Esto evidencia un desarrollo de la 
Volumen 47 (I), enero-junio $201 \mathrm{II}$

alfarería que tuvo su culminación en la década de 1980 con la promulgación de las leyes que prohibían la fabricación de objetos de uso doméstico bañados con plomo (Londoño, 2006). Es importante señalar que esta prohibición ya se había impuesto a inicios del siglo xIx, como lo evidencia la cédula real del 30 de noviembre de i8or que suscribió el virrey del Perú. La misma exhortaba a la regulación de los alfareros en aras de evitar "perjuicios que causan a la salud las vasijas de cobre, plomo, y estaño y las de barro mal vidriado [...]” (Matraya y Ricci, I8I9/I978, p. 478).

En resumen, lo que puede encontrarse es que durante parte del XVII, el xVIII y hasta mediados del xIx, las elites locales pudieron adquirir bienes importados desde fuentes de producción como Perú o Europa. Igualmente, que se siguieron manufacturando y consumiendo cerámicas indígenas, práctica que comenzó a generar fusiones tecnológicas como la construcción de vasijas enrolladas (una técnica indígena) que eran bañadas en plomo (una no indígena), o cerámicas burdas estampadas con cruces. Dentro de esta visión a gran escala, se puede apreciar que, a medida que se avanza en el tiempo, proliferan en el centro de Popayán y en los pueblos de indios los tipos locales vidriados con niveles estables de consumo de cerámica de tradición indígena. Este panorama cambia abruptamente finalizado el XIX.

Lo que resulta superfluo es señalar que la importación de loza evidencia la estructura jerárquica de la sociedad colonial. Esta aseveración, que es del todo cierta, borra fenómenos más complejos como las fusiones de tradiciones tecnológicas en la producción de vasijas asociadas a la ingesta de alimentos. Al parecer, lo que se fue configurando en el desarrollo de la cultura colonial en el Valle de Popayán fue una sociedad en la cual existía un sustrato del que se desprendían opciones discretas de accesos a diversos capitales. Este sustrato es lo que apropiadamente Hernán Torres (I999) definió, desde la antropología interpretativa, como la cultura tradicional de Popayán.

En suma, si los tipos importados evidencian un acceso privilegiado a ciertos ítems, lo cual es indicativo de una estructura social jerárquica, igualmente la permanencia de los tipos indígenas burdos, que se hallan tanto en el centro histórico como en las periferias, hablan de un sustrato común, lo cual cuestiona la idea de que primaba la diferenciación social. 
En sus trabajos etnográficos, Hernán Torres (1999) propone la existencia de una ideología de la ciudad en la cual los habitantes tratan de borrar las diferencias sociales a través de ciertos rituales como los de la Semana Santa. En este evento ritual, Torres encontró que los pobladores de la Popayán tradicional perciben la semana como el período en el cual el campo entra a la ciudad.

Por lo menos antes del terremoto de 1983, cuando la ciudad conservaba la fisonomía con la cual inició el siglo, en la semana mayor las comidas predilectas eran las tradicionales, que eran de amplio acceso. Es decir, que en el momento de más intensificación cultural los alimentos preferidos eran los elaborados en los pueblos de indios. Es posible visualizar esta tendencia cultural en el registro arqueológico en la medida en que, sin importar si es el centro histórico o la periferia, los tipos cerámicos predominantes son los de tradición indígena.

Es importante señalar que esta tendencia ha estado cambiando drásticamente en los últimos años, en especial por los procesos de patrimonialización que han venido rompiendo el sentido de comunitas, fundamental en la cultura tradicional de Popayán (cf. Torres, 1999).

\section{LO QUE DICEN LOS DATOS ETNOGRÁFICOS}

[l trabajo arqueológico previo hubiera podido terminar comprobando lo obvio: que las clases acaudaladas de Popayán -marcaban sus diferencias con el uso de platos, tazas y vasijas importadas. Menos obvio era señalar que desde los enterramientos de indígenas con peruleras, pasando por las cerámicas indígenas con incisiones de cruces, se fue formando una base que identificaba a los habitantes del Valle de Popayán con un sistema simbólico en el cual las diferencias sociales se entendían como pertenecientes a un sistema trascendente. En ese sentido, a pesar de las sujeciones y las desigualdades, existían espacios de encuentro asociados a festividades religiosas que invertían las relaciones sociales, lo que ha llevado a antropólogos como Hernán Torres a señalar que mejor que la metáfora de la pirámide social para aludir a las diferencias sociales en Popayán, es más adecuado representarlas como un arcoíris en el cual no se sabe dónde termina e inicia un color (Torres, 1999). 
Volumen 47 (I), enero-junio $20 I I$

En su análisis interpretativo, Hernán Torres se preguntó por la cultura tradicional de Popayán que sufrió un cambio abrupto como consecuencia del terremoto de I983. Para poder leer esta cultura, Torres tomó como texto la popular Torre del Reloj. A través de las imágenes que evocaba la Torre, este antropólogo encontró una marcada noción del tiempo en esta sociedad: a diferencia del tiempo moderno, que corre, en el mundo tradicional el tiempo anda (Torres, I999, p. I0).

En su lectura de la cultura tradicional de Popayán, Torres encontró que, en el momento en que las familias acaudaladas vivían en el centro de la ciudad en casas de dos plantas, existían sectores pobres de la sociedad que vivían en el primer piso y administraban pequeñas tiendas. En muchos casos, estas familias que manejaban las tiendas servían en la casa de los ricos. En esta interacción, se gestaban formas de amistad, en especial entre gente joven, de tal suerte que se generaba una apariencia de igualdad (Torres, I999, p. I6).

Como tuve oportunidad de conversar con gente de Popayán que desde hace más de treinta o cuarenta años viven en Cali, en la primera mitad del siglo xx era común que los niños de clase alta se rascaran las niguas (tunga penetrans) con los niños de las clases bajas. Lo curioso del caso es que muchos de los niños de clase alta no padecían esta infección. Valga señalar que las personas infectadas del parásito evitaban que los dedos de los pies tocaran el piso, por lo que adoptaban una forma específica de caminar que, junto a la hinchazón, hacía que fueran denominadas patojos, palabra que se usa como gentilicio de la gente de Popayán.

Igualmente, muchas de estas personas, que más que informantes fueron maestros y amigos, señalaron que dentro de sus familias conformadas por más de cinco hijos solo los últimos habían sido amamantados por la madre y los demás, por nanas, que en la mayoría de los casos eran indígenas o afrodescendientes.

Si bien el amamantamiento puede ser comprendido como otra forma de usurpación, estas prácticas generaron, más que un espacio de apariencia de igualdad, como lo señaló Torres, verdaderos campos de interconexión que llevaron no solo a alimentar seres humanos sino a crearlos, dando lugar a ese complejo sistema de parentesco paralelo de Popayán basado en paternidades ilegítimas y maternidades por adquisición. 
Simultáneamente, ese campo de interconexión se configuró en una gastronomía regional que incluye platos con maní, papa, ají, aguacate, lulo, maíz, limón, carne de res, cerdo y pollo, y cuyas mayores artífices eran las cocineras afro (Patiño, 2007). De los fogones de negro era de donde salían las maravillosas empanadas, marranitas, tamales, sancochos, luladas, masatos, champús y docenas de dulces de frutos tropicales que acompañaban las veladas de Nochebuena y Semana Santa de toda la base de la población y de su elite. Eso es precisamente lo que se refleja en el registro arqueológico.

Igualmente, como lo señaló Hernán Torres (1999), la cultura tradicional de Popayán sufrió una abrupta transformación a partir de un desarrollo no controlado de la ciudad como consecuencia de gran cantidad de recursos que ingresaron provenientes de la ayuda nacional e internacional por el terremoto de ig83. Aunque no hay estudios muy extensos al respecto, se sabe que, dado un precario control del uso del espacio en la década de I980, no fue posible tener un cálculo confiable de la población damnificada. Esta falla en el sistema de información permitió que un sinnúmero de inmigrantes de pueblos del departamento llegaran en busca de algún tipo de subsidio para la adquisición de viviendas.

En medio de esas dinámicas, el crecimiento demográfico de Popayán se hizo palpable en la década de r980 y allí jugó un papel importante la inyección de capitales. Dentro de este juego, el ingreso de cuantiosos recursos permitió la formación de cierto mercado, con lo cual se estimuló la llegada de bancos y se hizo más compleja la administración fiscal. Dentro de este proceso, las casas del centro de Popayán fueron abandonadas para dar paso a la apertura de corporaciones financieras que establecieron allí, en el centro de la ciudad, su centro de operación.

Paralelo al proceso de despoblamiento del centro histórico (lo cual rompió el modelo colonial de familias ricas que convivían con su servidumbre), se inició un proceso de formación de propiedad horizontal, sobre todo al norte de la ciudad, donde las nuevas configuraciones espaciales han inhibido las interrelaciones propias de la cultura tradicional de Popayán. En los nuevos apartamentos de tres habitaciones, hay algún recinto de dos metros por uno, al que le llaman cuarto de servicio.

Como siempre lo señaló Hernán Torres en diversas conferencias, la modernización en Popayán había llevado a una deshumanización que se podía apreciar en los inhabitables espacios 
Volumen 47 (I), enero-junio $20 I I$

donde la clase media confinaba a niñas campesinas, condenadas así a pasar su adolescencia sirviendo en un mundo sin interconexiones trascendentes. Esta nueva forma de esclavitud, que no tiene parangón con las relaciones de sujeción de la cultura colonial de Popayán (en las cuales había espacios de inversión que distensionaban el campo de sujeción), ha desembocado en formas aberrantes como el outsourcing étnico. Con ello se puede denominar la práctica de ofrecer, mediante oficinas laborales, "caucanas sumisas" para que limpien domicilios en ciudades como Cali. Dado que la compañía es la que consigue el cliente, estas empleadas caucanas reciben míseras sumas de dinero a cambio de extenuantes jornadas de trabajo. Lamentablemente sobre este fenómeno no hay estudios sistemáticos.

De tal suerte, en el caso de Popayán, la modernización ha desconfigurado el sistema de relaciones que unía diversos sectores de la sociedad. Prueba de estas formas de interconexión eran los objetos bañados de plomo que se usaban en todos los estratos sociales como también la cerámica de tradición indígena que hemos denominado Popayán Alisado Sin Pintura. La modernidad señaló fronteras más claras entre las clases sociales con las cuales se generó una presión demográfica que configuró los cordones de miseria alrededor de Popayán y un fortalecimiento de los colectivos étnicos en términos territoriales.

Un mapa del movimiento ocurrido en el Valle de Popayán podría mostrar que la delimitación de resguardos indígenas en las últimas cuatro décadas corre paralelo a procesos de formación de una ciudad que requiere extensas áreas para albergar a los medianos sectores de clase media y a los amplios sectores de clases bajas.

Se podría señalar que, como en ninguna época de la historia en la región, se han generado sendos abismos que imposibilitan el reconocimiento de los diferentes sectores sociales en una serie de prácticas cotidianas como alimentarse, vestirse, sentir nostalgia por un paisaje, como resultado del diálogo con ontologías locales.

Visibilizar estas interconexiones no intervenidas podría ser un buen recurso para una antropología que vaya más allá de la simple autocomplacencia de la globalización y de la comprobación de estratificaciones sociales. 
Arqueología histórica de Popayán y la visibilización de su cultura tradicional

\section{ReFerencias}

Barona, G. (I995). La maldición de Midas en una región del mundo colonial: Popayán 1730/I830. Cali: Universidad del Valle, Facultad de Humanidades. Historia y Sociedad.

Brizuela, Á. (2002). Las peruleras del pozo de Casas Terrin (Panamá La Vieja). Propuesta tipológica inicial de los bordes. Arqueología de Panamá La Vieja, 3, I34-I54.

Caicedo, A. (2006). Arqueología del contacto cultural en la Popayán colonial (siglos XVI y XVII). Tesis no publicada de Antropología, Universidad del Cauca, Colombia.

Cubillos, J. C. (I959). El Morro de Tulcán (pirámide prehispánica). Arqueología de Popayán, Cauca, Colombia. Revista Colombiana de Antropología, 8, 215-357.

Cubillos, J. C. (1984). Asentamientos prehispánicos en la suela plana del río Cauca. Bogotá: Fundación de Investigaciones Arqueológicas Nacionales, Banco de la República.

Deagan, K. (1987). Artifacts of the Spanish Colonies of Florida and the Caribbean, I500-I800. Ceramics, Glass, and Beads (Vol. I). Washington D. C.: Smithsonian Institution.

Fabian, J. (I983). Time and the Other: How Anthropology Makes Its Object. New York: Columbia University.

GNECCO, C. (I995). Praxis científica en la periferia: notas para una historia social de la arqueología colombiana. Revista Española de Antropología Americana, 25, 9-22.

Goggin, J. (1968). Spanish Majolica in the New World: Types of the Sixteenth to Eighteenth Centuries. New Haven: Yale University Press, Department of Anthropology.

Kendall, B. (I986). Bourbons and Brandy: Imperial Reform in EighteenthCentury Arequipa. Alburqueque: University of New Mexico Press.

LOBOGUERRERO, J. (200I/2002). Objetos cotidianos en la historia de la resistencia indígena en Colombia. Del documento de archivo al material arqueológico. Revista de Antropología y Arqueología, Universidad de los Andes, I3, 26-48.

LondoÑo, W. (2000). Reflexiones sobre un trabajo de campo en Novirao. Tesis no publicada de Antropología, Universidad del Cauca, Colombia.

Londoño, W. (2006). Hacia la identificación de patrones de consumo de cerámica vidriada y republicana. Bogotá: FIAN. Informe inédito. 
Volumen 47 (I), enero-junio 201

López, G. C. (I977). Breve noticia sobre la cerámica española. Boletín del Instituto Nacional de Antropología e Historia, México, 22, 37-59.

MAtraya y RicCi, J. J. (I8I9/I978). Catálogo cronológico de las pragmáticas, cédulas, decretos, órdenes y resoluciones reales generales emanados después de la recopilación de las leyes de Indias. Buenos Aires: Instituto de Investigaciones de Historia del Derecho.

Méndez, M. (2007). Historia y arqueología de la unidad conventual de San Francisco de Popayán. Popayán: Diseño Gráfico e Impresiones.

Patiño, G. (2007). Fogón de negros. Cocina y cultura en una región latinoamericana. Bogotá: Convenio Andrés Bello.

Polo, F. (2009). Identificación de atributos cerámicos de tradición indígena en cerámica de técnica prehispánica en el Valle de Popayán: hacia una arqueología de la cultura colonial. Tesis no publicada de Antropología, Universidad del Cauca, Colombia.

Reichel-Dolmatoff, G. (I950). Los kogi: una tribu de la Sierra Nevada de Santa Marta, Colombia (Tomo I). Revista del Instituto Etnológico Nacional, 4.

Reichel-Dolmatoff, G. (I95Ia). Datos histórico-culturales sobre las tribus de la antigua gobernación de Santa Marta. Bogotá: Banco de la República.

Reichel-Dolmatoff, G. (I95Ib). Los kogi: una tribu de la Sierra Nevada de Santa Marta, Colombia (Tomo II). Bogotá: Editorial Iqueima.

Reichel-Dolmatoff, G. (I953). Contactos y cambios culturales en la Sierra Nevada de Santa Marta. Revista Colombiana de Antropología, I, I5-I22.

Reichel-Dolmatoff, G. (I954). Investigaciones arqueológicas en la Sierra Nevada de Santa Marta (partes I y 2). Revista Colombiana de Antropología, 2, I45-206.

Reichel-Dolmatoff, G. (I997). Arqueología de Colombia. Un texto introductorio. Bogotá: Biblioteca de la Presidencia de la República.

Rice, P. (I997). Wine and Brandy Production in Colonial Peru: A Historical and Archaeological Investigation. Journal of Interdisciplinary History, 27, 455-479.

Rice, P. \& Beck, S. van. (I990). The Spanish Colonial Kiln Tradition of Moquegua, Perú. Historical Archaeology, 27 (4), 65-8I.

Rice, P. \& Smith, G. (I989). The Spanish Colonial Wineries of Moquegua, Perú. Historical Archaeology, 23 (2), 4I-49. 
Arqueología histórica de Popayán y la visibilización de su cultura tradicional

RovirA, B. (I997). Hecho en Panamá: la manufactura colonial de mayólicas. Revista Nacional de Cultura, Panamá, 27, 67-85.

RoviRA, B. (2002). Presencia de mayólicas panameñas en el mundo colonial. Algunas consideraciones acerca de su distribución y cronología. Arqueología de Panamá La Vieja, 3, II2-I33.

SERULniKov, S. (2006). Conflictos sociales e insurrección en el mundo colonial andino. El norte de Potosí en el siglo XVIII. Buenos Aires: Fondo de Cultura Económica.

Schávelzon, D. (200I). Catálogo de cerámicas históricas de Buenos Aires (siglos XVI-XX). Con notas sobre la región del Río de la Plata. Buenos Aires: Faar, Telefónica, Fadu, Cau.

Therrien, M., Uprimy, E., Lobo-Guerrero, J., Salamanca, M., Gattán, F. \& Fandiño, M. (2002). Catálogo de cerámica colonial y republicana de la Nueva Granada: producción local y materiales foráneos (costa caribe, altiplano cundiboyacense, Colombia). Bogotá: Fundación de Investigaciones Arqueológicas Nacionales, Banco de la República.

Torres, H. (I999). Alegorías, metáforas y textos etnográficos: la Torre del Reloj de Popayán, Cuadernos de Antropología Poética, I (I), I-36.

URDANETA, M. (I99I). Huellas de pishau en el resguardo de Guambía. Boletín del Museo del Oro, 3I, 3-30. 